\title{
EXTRINSIC CIRCULAR TRAJECTORIES ON TOTALLY $\eta$-UMBILIC REAL HYPERSURFACES IN A COMPLEX HYPERBOLIC SPACE
}

\author{
TUYa BAO AND TOSHIAKI AdACHI
}

\begin{abstract}
A trajectory for a Sasakian magnetic field, which is a generalization of geodesics, on a real hypersurface in a complex hyperbolic space $\mathbf{C} H^{n}$ is said to be extrinsic circular if it can be regarded as a circle as a curve in $\mathbf{C} H^{n}$. We study how the moduli space of extrinsic circular trajectories, which is the set of their congruence classes, on a totally $\eta$-umbilic real hypersurface is contained in the moduli space of circles in $\mathbf{C} H^{n}$. From this aspect we characterize tubes around totally geodesic complex hypersurfaces $\mathbf{C} H^{n-1}$ in $\mathrm{CH}^{n}$ by some properties of such trajectories.
\end{abstract}

\section{Introduction}

In their paper [12] Kajiwara and Maeda gave several characterizations of nonnegatively curved geodesic spheres in a complex hyperbolic space. Among them the authors are interested in the characterization of geodesic spheres by using extrinsic circular geodesics. A smooth curve $\sigma$ parameterized by its arclength on a Riemannian manifold is said to be a circle of geodesic curvature $k(\geq 0)$ if it satisfies the system of differential equations $\nabla_{\dot{\sigma}} \dot{\sigma}=k Y, \nabla_{\dot{\sigma}} Y=-k \dot{\sigma}$ with a field $Y$ of unit vectors along $\sigma$. Equivalently, a smooth curve $\sigma$ parameterized by its arclength is a circle if it satisfies $\nabla_{\dot{\sigma}} \nabla_{\dot{\sigma}} \dot{\sigma}=-k^{2} \dot{\sigma}$. When $k=0$, it is a geodesic. Hence we may say that circles are simplest curves next to geodesics from the viewpoint of Frenet formula. A curve on a submanifold is said to be extrinsic circular if it is a circle of positive geodesic curvature as a curve in the ambient space. Thus Kajiwara and Maeda noted when geodesics on a real hypersurface can be seen as "nice" curves in its ambient space.

2000 Mathematics Subject Classification. Primary 53C22, Secondary 53B35.

Key words and phrases. Sasakian magnetic fields, extrinsic circular trajectories, moduli space of circles, geodesic spheres, tubes around totally geodesic complex hypersurfaces.

The first author is partially supported by the Scientific Research Foundation of Inner Mongolia University for the Nationalities (No. NMDGP1416) and by the National Natural Science Foundation of China (No. 11561052).

The second author is partially supported by Grant-in-Aid for Scientific Research (C) (No. 24540075 and No. 16K05126) Japan Society for the Promotion of Science.

Received February 5, 2016. 
One can easily guess that some properties of extrinsic shapes of some curves on a submanifold show properties of the embedding. For example, extrinsic shapes of geodesics on a standard sphere in a Euclidean space are circles, and this property characterize a standard sphere among hypersurfaces in a Euclidean space. From this point of view Kajiwara and Maeda showed that a connected real hypersurface in a complex hyperbolic space $\mathbf{C H}^{n}$ is a geodesic sphere of positive sectional curvature if geodesics orthogonal to the characteristic vector are circles in $\mathbf{C} H^{n}$ of geodesic curvature greater than the square root of holomorphic sectional curvature of $\mathbf{C} H^{n}$ (see $\$ 2$ for more precisely).

Being inspired by this result we study extrinsic shapes of trajectories for Sasakian magnetic fields on totally $\eta$-umbilic real hypersurfaces in $\mathbf{C} H^{n}$, which are geodesic spheres, horospheres and tubes around totally geodesic complex hypersurfaces $\mathbf{C} H^{n-1}$. A Sasakian magnetic field is a constant multiple of the canonical 2-form $\mathbf{F}_{\phi}$ obtained by the characteristic tensor $\phi$ of a real hypersurface in a Kähler manifold (see §4). For a Sasakian magnetic field $\mathbf{F}_{\kappa}=\kappa \mathbf{F}_{\phi}$, a smooth curve $\gamma$ parameterized by its arclength is said to be its trajectory if it satisfies the differential equation $\nabla_{\dot{\gamma}} \dot{\gamma}=\kappa \phi \dot{\gamma}$. When $\kappa=0$ it is nothing but a geodesic. Hence, trajectories for Sasakian magnetic fields on real hypersurfaces are generalization of geodesics. Since trajectories are closely related with the almost contact metric structure of the underlying real hypersurface, the authors think that their properties show more properties of the real hypersurface than geodesics. In this paper, we refine the study on extrinsic circular trajectories for Sasakian magnetic fields on totally $\eta$-umbilic real hypersurfaces in $\mathbf{C} H^{n}$ in [5]. We study the behavior of the set of congruence classes of these trajectories on each totally $\eta$-umbilic real hypersurface in the moduli space of circles, which is the set of all congruence classes of circles, on $\mathbf{C H}^{n}$. We show that moduli spaces of extrinsic circular trajectories on geodesic spheres form a foliation on the moduli space of bounded circles on $\mathrm{CH}^{n}$. Also, in view of those moduli spaces we characterize tubes around totally geodesic $\mathbf{C} H^{n-1}$ in $\mathbf{C H}$.

\section{Supplemental results for Kajiwara-Maeda's characterization}

In order to make clear our standing point of studies, we shall start by recalling a characterization of geodesic spheres of small radius given by Kajiwara and Maeda [12], and by giving its supplemental results.

On a real hypersurface $M$ of a Kähler manifold $\tilde{M}$, we have a canonical contact metric structure which is induced by the complex structure $J$ on $\tilde{M}$. For a (local) unit normal vector field $\mathscr{N}$ on $M$ in $\tilde{M}$ and the Riemannian metric $\langle$, on $\tilde{M}$, we define a vector field $\xi$ by $\xi=-J \mathscr{N}$, a 1 -form $\eta$ by $\eta(v)=\langle v, \xi\rangle$ and a $(1,1)$-tensor field $\phi$ by $\phi(v)=J v-\eta(v) \mathscr{N}$ for each tangent vector $v \in T M$. With the induced metric $\langle$,$\rangle on M$ we have a contact metric structure $(\phi, \xi, \eta,\langle\rangle$, on $M$. We call $\xi$ the characteristic vector field and $\phi$ the characteristic tensor on $M$.

Let $l: M \rightarrow \tilde{M}$ denote an isometric embedding of a real hypersurface $M$ into the ambient space $\tilde{M}$. For a smooth curve $\gamma$ on $M$, we can regard it as a curve 
in $\tilde{M}$ by considering $\imath \circ \gamma$ and call it the extrinsic shape of $\gamma$. For the sake of simplicity, we usually denote the extrinsic shape $l \circ \gamma$ also by $\gamma$. In their paper [12] Kajiwara and Maeda characterized geodesic spheres of nonnegative curvature by a property of extrinsic shapes of geodesics.

Theorem (Kajiwara-Maeda [12] (cf. [16])). Let $M$ be a connected real hypersurface in a complex hyperbolic space $\mathbf{C} H^{n}(c)$ of constant holomorphic sectional curvature $c$. Then the following conditions are mutually equivalent:

(1) $M$ is locally congruent to a geodesic sphere all of whose sectional curvatures are nonnegative;

(2) $M$ is locally congruent to a geodesic sphere $G(r)$ of radius $r \leq(\log 3) /$ $\sqrt{|c|}$

(3) At each point $p \in M$ there exist a constant $k_{p}$ with $k_{p} \geq \sqrt{|c|}$ and orthonormal vectors $v_{1}, \ldots, v_{2 n-2} \in U_{p} M$ orthogonal to $\xi_{p}$ satisfying that the extrinsic shape of each geodesic $\gamma_{i}$ on $M$ with $\dot{\gamma}_{i}(0)=v_{i}$ is a circle of geodesic curvature $k_{p}$.

Since they restricted themselves to real hypersurfaces of nonnegative curvature, they showed the above result. But their proof tells more. We consider the following condition:

(ES) At each point $p \in M$ of a real hypersurface $M$ in $\mathbf{C} H^{n}$, there exist linearly independent unit tangent vectors $v_{1}, \ldots, v_{2 n-2} \in U_{p} M$ orthogonal to $\xi_{p}$ satisfying that the extrinsic shapes of geodesic $\gamma_{i}$ $(i=1, \ldots, 2 n-2)$ on $M$ with $\dot{\gamma}_{i}(0)=v_{i}$ are circles of common positive geodesic curvature $k_{p}$.

We note that Condition (ES) is a bit weaker than the condition in the third condition in Kajiwara-Maeda's result, because we need not assume that $v_{1}, \ldots, v_{2 n-2} \in U_{p} M$ are orthogonal. We have the following.

Proposition 1. A connected real hypersurface $M$ in $\mathbf{C} H^{n}(c)$ is locally congruent to a geodesic sphere $G(r)$ of radius $r \geq(\log 3) / \sqrt{|c|}$ if and only if it satisfies Condition (ES) and there is a point $p_{0} \in M$ with $\sqrt{|c|} / 2<k_{p_{0}} \leq \sqrt{|c|}$.

Proposition 2. A connected real hypersurface $M$ in $\mathbf{C} H^{n}(c)$ is locally congruent to a horosphere HS if and only if it satisfies Condition (ES) and there is a point $p_{0} \in M$ with $k_{p_{0}}=\sqrt{|c|} / 2$.

Proposition 3. A connected real hypersurface $M$ in $\mathbf{C H}^{n}(c)$ is locally congruent to a tube $T(r)$ of radius $r$ around totally geodesic $\mathbf{C H} H^{n-1}$ if and only if it satisfies Condition (ES) and there is a point $p_{0} \in M$ with $k_{p_{0}}<\sqrt{|c|} / 2$.

Since one can easily show the above results along the same lines as in the proof of Kajiwara-Maeda's result, we here give outlines. We denote by $A_{M}$ the shape operator of $M$ in $\mathbf{C} H^{n}$ associated with a unit normal $\mathscr{N}$. When the characteristic vector field of a real hypersurface is principal at each point, it is 
said to be a Hopf hypersurface. Its principal curvatures satisfy the following properties.

Lemma $1([14,13])$. Let $M$ be a Hopf real hypersurface in $\mathbf{C} H^{n}(c)$. Then its principal curvatures satisfy the following.

(1) The principal curvature $\delta_{M}$ associated with $\xi$ is locally constant.

(2) If a nonnull vector $v \in T M$ satisfies $A_{M} v=\lambda v$, then $\left(2 \lambda-\delta_{M}\right) A_{M} \phi v=$ $\left(\delta_{M} \lambda+(c / 2)\right) \phi v$ holds.

Under Condition (ES), we find that either $A_{M} v_{i}=k_{p} v_{i}$ or $A_{M} v_{i}=-k_{p} v_{i}$ holds for $i=1, \ldots, 2 n-2$. This shows that $v_{i}$ and $\xi_{p}$ are principal curvature vectors, and in particular that $M$ is a Hopf real hypersurface. Applying Lemma 1 we can conclude that $M$ has at most three constant principal curvatures $k,-k$ and $\delta_{M}$, where, $\delta_{M}$ denotes the principal curvature associated with the characteristic vector field. As connected Hopf real hypersurfaces in $\mathbf{C} H^{n}(c)$ all of whose principal curvatures are constant are classified by Berndt [9], considering their principal curvatures we get the results. Here, such hypersurfaces are horospheres $H S$, geodesic spheres $G(r)$ of radius $r$, tubes $T(r)$ of radius $r$ around totally geodesic $\mathbf{C} H^{n-1}$, tubes $T_{\ell}(r)(\ell=1, \ldots, n-2)$ of radius $r$ around totally geodesic $\mathbf{C} H^{\ell}$, and tubes $R(r)$ of radius $r$ around totally geodesic real hypersurface $\mathbf{R} H^{n}$. When a real hypersurface $M$ is one of $H S, G(r)$ and $T(r)$ it has two principal curvatures;

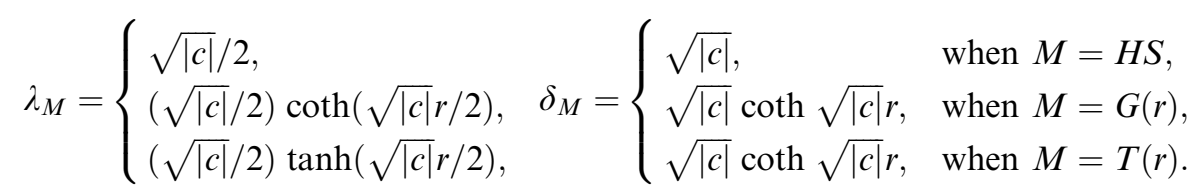

These horospheres, geodesic spheres and tubes around totally geodesic complex hypersurfaces are called totally $\eta$-umbilic real hypersurfaces because all tangent vectors orthogonal to their characteristic vectors are principal. When $M$ is either $T_{\ell}(r)$ or $R(r)$ it has two principal curvatures;

$$
\lambda_{M}=(\sqrt{|c|} / 2) \operatorname{coth}(\sqrt{|c|} r / 2), \quad \mu_{M}=(\sqrt{|c|} / 2) \tanh (\sqrt{|c|} r / 2)
$$

for vectors orthogonal to the characteristic vector and

$$
\delta_{M}= \begin{cases}\sqrt{|c|} \operatorname{coth} \sqrt{|c|} r, & \text { when } M=T_{\ell}(r), \\ \sqrt{|c|} \tanh \sqrt{|c|} r, & \text { when } M=R(r) .\end{cases}
$$

\section{Circles on a complex hyperbolic space}

By results in the previous section, it seems there is a relationship between geodesic curvatures of circles on $\mathrm{CH}^{n}$ and its real hypersurfaces. In order to study this we here review some basic properties of circles on $\mathbf{C} H^{n}$. 
Two smooth curves $\sigma_{1}, \sigma_{2}$ on a Riemannian manifold $N$ parameterized by their arclengths are said to be congruent to each other if there is an isometry $\varphi$ of $N$ and a constant $t_{0}$ satisfying $\sigma_{2}\left(t+t_{0}\right)=\varphi \circ \sigma_{1}(t)$ for all $t$. Being different from circles on a Euclidean space or on a real hyperbolic space, even if two circles on $\mathbf{C} H^{n}(c)$ have the same geodesic curvatures they are not necessarily congruent to each other. For a circle $\sigma$ of positive geodesic curvature on $\mathbf{C} H^{n}(c)$ which satisfies $\nabla_{\dot{\sigma}} \dot{\sigma}=k_{\sigma} Y, \nabla_{\dot{\sigma}} Y=-k_{\sigma} \dot{\sigma}$ with a field $Y$ of unit vectors along $\sigma$, we define its complex torsion $\tau_{\sigma}$ by $\tau_{\sigma}=\langle\dot{\sigma}, J Y\rangle$ with complex structure $J$ on $\mathbf{C} H^{n}$. Since $J$ is parallel, its complex torsion $\tau_{\sigma}$ is constant along $\sigma$ and satisfies $\left|\tau_{\sigma}\right| \leq 1$. It is known that two circles $\sigma_{1}, \sigma_{2}$ on $\mathbf{C} H^{n}(c)$ are congruent to each other if and only if $k_{\sigma_{1}}=k_{\sigma_{2}}$ and $\left|\tau_{\sigma_{1}}\right|=\left|\tau_{\sigma_{2}}\right|$ (see [17]). Therefore, the moduli space $\mathscr{M}_{2}\left(\mathbf{C} H^{n}\right)$ of circles of positive geodesic curvature, which is the set of all congruence classes of such circles, on $\mathbf{C H}^{n}$ is set theoretically identified with a band $(0, \infty) \times[0,1]$.

As $\mathbf{C} H^{n}$ is a Hadamard manifold, a simply connected complete manifold of nonpositive curvature, we can consider its ideal boundary $\partial \mathbf{C} H^{n}$. We say a smooth curve $\sigma: \mathbf{R} \rightarrow \mathbf{C} H^{n}$ to be unbounded in both directions, if both of the sets $\{\sigma(t) \mid t \leq 0\}$ and $\{\sigma(t) \mid t \geq 0\}$ are unbounded. For such a curve $\sigma$ we set $\sigma(-\infty)=\lim _{t \rightarrow-\infty} \sigma(t), \sigma(\infty)=\lim _{t \rightarrow \infty} \sigma(t)$ if they exist in $\partial \mathbf{C} H^{n}$, and call them its points at infinity. If we define a function $v:(0, \infty) \rightarrow[0,1]$ by

$$
v(k)= \begin{cases}0, & \text { if } 0<k<\sqrt{|c|} / 2, \\ \left(4 k^{2}+c\right)^{3 / 2} /(3 \sqrt{3}|c| k), & \text { if } \sqrt{|c|} / 2 \leq k \leq \sqrt{|c|}, \\ 1, & \text { if } k>\sqrt{|c|},\end{cases}
$$

we can describe properties of circles on $\mathbf{C H}^{n}(c)$ as follows ([6]):

1) A circle $\sigma$ is bounded if and only if either $k_{\sigma}>\sqrt{|c|}$ or $\tau_{\sigma}<v\left(k_{\sigma}\right)$;

2) A circle $\sigma$ is unbounded in both directions and has a single point at infinity $(\sigma(-\infty)=\sigma(\infty))$ if and only if $\sqrt{|c|} / 2 \leq k_{\sigma} \leq \sqrt{|c|}$ and $\left|\tau_{\sigma}\right|=v\left(k_{\sigma}\right)$;

3) A circle $\sigma$ is unbounded in both directions and has two distinct points at infinity if and only if either $k_{\sigma}<\sqrt{|c|} / 2$ or $\sqrt{|c|} / 2 \leq k_{\sigma} \leq \sqrt{|c|}$ and $\left|\tau_{\sigma}\right|>v\left(k_{\sigma}\right)$

4) Every bounded circle $\sigma$ with $\tau_{\sigma}=0$ is closed of length $4 \pi / \sqrt{4 k^{2}+c}$;

5) Every bounded circle $\sigma$ with $\tau_{\sigma}= \pm 1$ is closed of length $2 \pi / \sqrt{k^{2}+c}$.

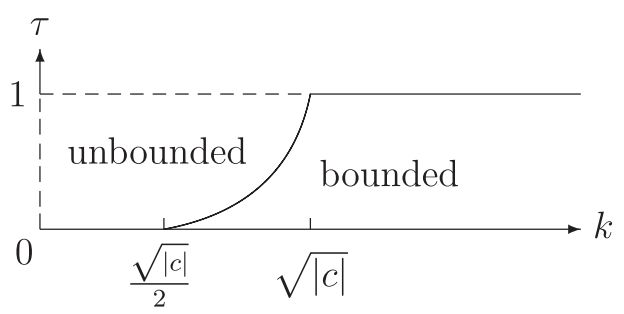

Figure 1. The moduli space $\mathscr{M}_{2}\left(\mathbf{C} H^{n}\right)$ of circles on $\mathbf{C} H^{n}(c)$ 
We denote by $\nabla$ and $\tilde{\nabla}$ the Riemannian connections on a real hypersurface $M$ and on $\mathbf{C} H^{n}(c)$, respectively. We recall that Gauss and Weingarten formulae are given as $\tilde{\nabla}_{X} Y=\nabla_{X} Y+\left\langle A_{M} X, Y\right\rangle \mathscr{N}$ and $\tilde{\nabla}_{X} \mathscr{N}=-A_{M} X$ for vector fields $X, Y$ tangent to $M$. We take a geodesic $\gamma$ on $M$ whose initial vector is orthogonal to the characteristic vector. By use of Gauss formula, if the extrinsic shape of $\gamma$ is a circle, then its complex torsion is null. Thus we may say that Kajiwara-Maeda's result and its supplements show a relationship between geodesic curvatures of circles of null complex torsion and real hypersurfaces from the viewpoint of extrinsic shapes of geodesics. We are hence interested in some relativity of real hypersurfaces and circles of other complex torsions.

\section{Extrinsic shapes of trajectories for Sasakian magnetic fields}

Let $M$ be a real hypersurface in $\mathbf{C} H^{n}(c)$. We here extend the notion of geodesics on $M$. By using the characteristic tensor $\phi$ we define a 2 -form $\mathbf{F}_{\phi}$ on $M$ by $\mathbf{F}_{\phi}(u, . v)=\langle u, \phi v\rangle$. Since it is a closed 2-form (see [8]), we say its constant multiples to be Sasakian magnetic fields. Generally, a closed 2-form on a Riemannian manifold is called a magnetic field because it can be regarded as a generalization of a static magnetic field on a Euclidean 3-space (see [10, 18] and also [1], for example). A smooth curve $\gamma$ on $M$ parameterized by its arclength is said to be a trajectory for a Sasakian magnetic field $\mathbf{F}_{\kappa}=\kappa \mathbf{F}_{\phi}$ if it satisfies $\nabla_{\dot{\gamma}} \dot{\gamma}=\kappa \phi \dot{\gamma}$. As trajectories for $\mathbf{F}_{0}$ are geodesics, we can consider trajectories as perturbations of geodesics.

We here consider trajectories for Sasakian magnetic fields whose extrinsic shapes are circles. Given a trajectory $\gamma$ for $\mathbf{F}_{\kappa}$, we define its structure torsion $\rho_{\gamma}$ by $\rho_{\gamma}=\left\langle\dot{\gamma}, \xi_{\gamma}\right\rangle$, where $\xi$ denotes the characteristic vector of $M$. Since Gauss formula and Weingarten formula lead us to $\nabla_{X} \xi=\phi A_{M} X$, we have

$$
\rho_{\gamma}^{\prime}=\nabla_{\dot{\gamma}}\left\langle\dot{\gamma}, \xi_{\gamma}\right\rangle=\left\langle\kappa \phi \dot{\gamma}, \xi_{\gamma}\right\rangle+\left\langle\dot{\gamma}, \phi A_{M} \dot{\gamma}\right\rangle=\frac{1}{2}\left\langle\dot{\gamma},\left(\phi A_{M}-A_{M} \phi\right) \dot{\gamma}\right\rangle,
$$

because the shape operator $A_{M}$ is symmetric and the characteristic tensor $\phi$ is anti-symmetric. Thus $\rho_{\gamma}$ is constant along $\gamma$ if $A_{M}$ and $\phi$ are simultaneously diagonalizable $\left(A_{M} \phi=\phi A_{M}\right)$. It is known that such a property holds for geodesic spheres $G(r)$, horospheres $H S$, and tubes $T(r)$ and $T_{\ell}(r)$ around totally geodesic $\mathbf{C H}^{\ell}(\ell=1, \ldots, n-1)$, which are usually called real hypersurfaces of type (A).

We now restrict ourselves to $G(r), H S$ and $T(r)$ in $\mathbf{C} H^{n}(c)$. For a trajectory $\gamma$ for $\mathbf{F}_{\kappa}$ on such a real hypersurface $M$, by using Gauss and Weingarten formulae we have

$$
\left\{\begin{array}{l}
\tilde{\nabla}_{\dot{\gamma}} \dot{\gamma}=\kappa \phi \dot{\gamma}+\left(\lambda_{M}\left(1-\rho_{\gamma}^{2}\right)+\delta_{M} \rho_{\gamma}^{2}\right) \mathscr{N} \\
\tilde{\nabla}_{\dot{\gamma}}\left[\kappa \phi \dot{\gamma}+\left(\lambda_{M}\left(1-\rho_{\gamma}^{2}\right)+\delta_{M} \rho_{\gamma}^{2}\right) \mathscr{N}\right] \\
=-\left\{\kappa^{2}\left(1-\rho_{\gamma}^{2}\right)+\left(\lambda_{M}+\left(\delta_{M}-\lambda_{M}\right) \rho_{\gamma}^{2}\right)^{2}\right\} \dot{\gamma} \\
\quad+\left(\lambda_{M}-\kappa \rho_{\gamma}+\left(\delta_{M}-\lambda_{M}\right) \rho_{\gamma}^{2}\right)\left(\kappa+\left(\delta_{M}-\lambda_{M}\right) \rho_{\gamma}\right)\left(\rho_{\gamma} \dot{\gamma}-\xi_{\gamma}\right) .
\end{array}\right.
$$


Thus we get conditions that the extrinsic shapes of trajectories are circles (cf. $[3,5])$.

Lemma 2. A trajectory $\gamma$ for $\mathbf{F}_{\kappa}$ on a real hypersurface $M$ which is congruent to one of $G(r)$, HS and $T(r)$ in $\mathbf{C} H^{n}(c)$ is extrinsic circular if and only if it satisfies one of the following conditions:

1) $\rho_{\gamma}= \pm 1$,

2) $\lambda_{M}-\kappa \rho_{\gamma}+\left(\delta_{M}-\lambda_{M}\right) \rho_{\gamma}^{2}=0$,

3) $\kappa+\left(\delta_{M}-\lambda_{M}\right) \rho_{\gamma}=0$.

Corresponding to each case, the geodesic curvature $k_{\gamma}$ and the complex torsion $\tau_{\gamma}$ of the extrinsic shape of $\gamma$ are as follows:

1) $k_{\gamma}=\delta_{M}, \tau_{\gamma}=\mp 1$,

2) $k_{\gamma}=|\kappa|, \tau_{\gamma}=-\operatorname{sgn}(\kappa)$,

3) $k_{\gamma}=\sqrt{\kappa^{2}-2 \lambda_{M} \kappa \rho_{\gamma}+\lambda_{M}^{2}}, \tau_{\gamma}=\left(2 \kappa \rho_{\gamma}^{2}-\kappa-\lambda_{M} \rho_{\gamma}\right) / k_{\gamma}$.

When $M$ is one of $G(r), H S$ and $T(r)$, we have $\delta_{M}-\lambda_{M}=|c| /\left(4 \lambda_{M}\right)$. Thus in the case $4 \kappa \lambda_{M}+|c| \rho_{\gamma}=0$, which is the third case of the above lemma, we can express the geodesic curvature and the complex torsion of the extrinsic shape as

$$
k_{\gamma}=\sqrt{\lambda_{M}^{2}+\frac{|c| \rho_{\gamma}^{2}}{2}+\frac{c^{2} \rho_{\gamma}^{2}}{16 \lambda_{M}^{2}}}, \quad \tau_{\gamma}=\frac{\rho_{\gamma}\left(|c|-2|c| \rho_{\gamma}^{2}-4 \lambda_{M}^{2}\right)}{4 k_{\gamma} \lambda_{M}} .
$$

First we study extrinsic circular trajectories for Sasakian magnetic fields on a given totally $\eta$-umbilic real hypersurface $M$. We denote by $\mathscr{E}(M)$ the set of all congruence classes of extrinsic circular trajectories on $M$ of the third type in Lemma 2. Since each isometry $\varphi$ of $M$ is equivariant, that is, there is an isometry $\tilde{\varphi}$ of $\mathbf{C} H^{n}$ satisfying $l \circ \varphi=\tilde{\varphi} \circ l$ with the isometric embedding $l: M \rightarrow$ $\mathbf{C} H^{n}$, we find that if two trajectories on $M$ are congruent to each other then their extrinsic shapes are congruent to each other in $\mathbf{C} H^{n}$. We can show the converse holds (see [2, 7]). Thus we can identify the set $\mathscr{E}(M)$ with the set of all congruence classes of circles which are extrinsic shapes of trajectories of the third type in Lemma 2 on $M$.

As the structure torsion of each trajectory satisfies $\left|\rho_{\gamma}\right| \leq 1$, for an extrinsic circular trajectory $\gamma$ of the third type in Lemma 2 on $M$, by the first equality of (4.2) we have $\lambda_{M} \leq k_{\gamma} \leq \lambda_{M}+\left(|c| /\left(4 \lambda_{M}\right)\right)$. By substituting the first equality of (4.2) into the second we obtain

$$
\tau_{\gamma}^{2}=\frac{\left(k_{\gamma}^{2}-\lambda_{M}^{2}\right)\left(32 \lambda_{M}^{2} k_{\gamma}^{2}+4 c \lambda_{M}^{2}-c^{2}\right)^{2}}{|c|\left(8 \lambda_{M}^{2}-c\right)^{3} k_{\gamma}^{2}} .
$$

If we regard the right hand side of (4.3) as a function $g(K)=g\left(K ; \lambda_{M}\right)$ on $K=k_{\gamma}^{2}$, we have

$$
\frac{d g}{d K}=\frac{\lambda_{M}^{2}(8 K-c)\left(8 K-4 \lambda^{2}+c\right)\left(32 \lambda_{M}^{2} K+4 c \lambda_{M}^{2}-c^{2}\right)}{|c|\left(8 \lambda_{M}^{2}-c\right)^{3} K^{2}} .
$$


We hence get the following:

1) When $M$ is either $H S$ or $G(r)$, then $g$ is monotone increasing with respect to $k_{\gamma}$, and $g\left(\lambda_{M}^{2}\right)=0, g\left(\left(\lambda_{M}+\frac{|c|}{4 \lambda_{M}}\right)^{2}\right)=1$.

2) When $M$ is $T(r)$, then $g$ is monotone increasing in the union of intervals

$$
\left(\lambda_{M}^{2},\left(4 \lambda_{M}^{2}-c\right) / 8\right] \cup\left[\left(-4 c+c^{2} \lambda_{M}^{-2}\right) / 32,\left(\lambda_{M}+\frac{|c|}{4 \lambda_{M}}\right)^{2}\right],
$$

and is monotone decreasing in the interval

$$
\left[\left(4 \lambda_{M}^{2}-c\right) / 8,\left(-4 c+c^{2} \lambda_{M}^{-2}\right) / 32\right]
$$

At the ends of these intervals we have

$$
\begin{aligned}
& g\left(\lambda_{M}^{2}\right)=g\left(\left(-4 c+c^{2} \lambda_{M}^{-2}\right) / 32\right)=0, \\
& g\left(\left(\lambda_{M}+\frac{|c|}{4 \lambda_{M}}\right)^{2}\right)=1, \quad g\left(\left(4 \lambda_{M}^{2}-c\right) / 8\right)=\frac{-\left(4 \lambda_{M}^{2}+c\right)^{3}\left(4 \lambda_{M}^{2}-c\right)}{|c|\left(8 \lambda_{M}^{2}-c\right)^{3}}(<1) .
\end{aligned}
$$

We note that $\lambda_{M}+\left(|c| /\left(4 \lambda_{M}\right)\right)=\delta_{M}$. Moreover, when $M=T(r)$ we have

1) $\sqrt{\left(4 \lambda_{M}^{2}-c\right) / 8}<\sqrt{|c|} / 2<\sqrt{\left(-4 c+c^{2} \lambda_{M}^{-2}\right) / 32}$

2) $\sqrt{\left(-4 c+c^{2} \lambda_{M}^{-2}\right) / 32}<\sqrt{|c|}$ if and only if $r>(2 \log (\sqrt{7}+1)-\log 6) / \sqrt{|c|}$,

3) $g\left(\left(4 \lambda_{M}^{2}-c\right) / 8\right)$ is monotone decreasing with respect to $\lambda_{M}$.

By the above argument, we find that the moduli space $\mathscr{E}(M)$ of extrinsic circular trajectories of the third type in Lemma 2 on $M$ is a curve on the moduli space $\mathscr{M}_{2}\left(\mathbf{C} H^{n}\right)$ of circles like Figures 2 and 3. Also, we find that the congruence class of extrinsic circular trajectories of the first type in Lemma 2 on $M$, which is expressed as $\left\{\left(\delta_{M}, 1\right)\right\}$ in $\mathscr{M}_{2}\left(\mathbf{C} J^{n}\right)=(0, \infty) \times[0,1]$, is contained in $\mathscr{E}(M)$. Thus, the moduli space of all extrinsic circular trajectories on $M$ is $\mathscr{E}(M) \cup\{(k, 1) \mid k>0\}$.

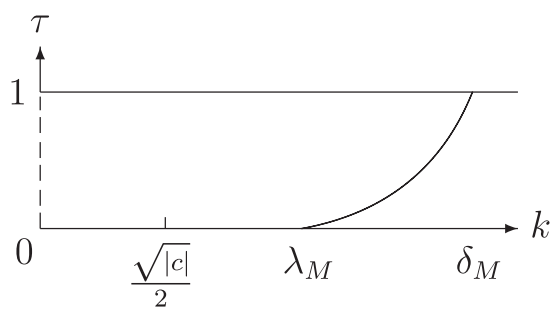

FIGURE 2. $\mathscr{E}(M)$ for $M=G(r)$

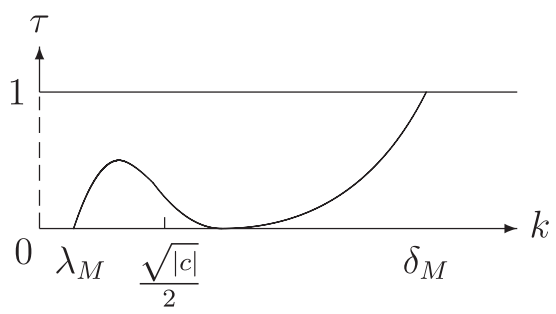

FIGURE 3. $\mathscr{E}(M)$ for $M=T(r)$ 
Next we study how the curve $\mathscr{E}(M)$ for a totally $\eta$-umbilic real hypersurface $M$ behaves with respect to the radius. We regard the right hand side of (4.3) as a function $h(\Lambda)=h\left(\Lambda ; k_{\gamma}\right)$ on $\Lambda=\lambda_{M}^{2}$. We have

$$
\frac{d h}{d \Lambda}=-\frac{\left(8 k_{\gamma}^{2}-c\right)^{2}\left(32 k_{\gamma}^{2} \Lambda+4 c \Lambda-c^{2}\right)(4 \Lambda+c)}{|c|(8 \Lambda-c)^{4} k_{\gamma}^{2}} .
$$

When $M=G(r)$, as $k_{\gamma} \geq \lambda_{M}>\sqrt{|c|} / 2$, we see $32 k_{\gamma}^{2} \Lambda+4 c \Lambda-c^{2}>0$ and $4 \lambda_{M}^{2}+c>0$. Hence we find that $d h / d \Lambda<0$ for $\lambda_{M}<k_{\gamma}<\delta_{M}$. Since $(\sqrt{|c|} / 2) \operatorname{coth}(\sqrt{|c|} r / 2)$ is monotone decreasing with respect to $r$, if we take two geodesic spheres $M=G\left(r_{1}\right), M^{\prime}=G\left(r_{2}\right)\left(r_{1}<r_{2}\right)$, the curves $\mathscr{E}(M)$ and $\mathscr{E}\left(M^{\prime}\right)$ do not have intersections (see Figure 4). Moreover, as $\lim _{r \rightarrow \infty} \lambda_{G(r)}=\sqrt{|c|} / 2$, $\lim _{r \rightarrow \infty} \delta_{G(r)}=\sqrt{|c|}$ and $\lim _{r \downarrow 0} \lambda_{G(r)}=\lim _{r \downarrow 0} \delta_{G(r)}=\infty$, we find that the moduli space $\mathscr{M}_{2}\left(\mathbf{C} H^{n}\right)$ of bounded circles on $\mathbf{C} H^{n}$ is covered by $\{\mathscr{E}(G(r)) \mid r>0\}$.

When $M=T(r)$, as $\lambda_{M}<\sqrt{|c|} / 2$, we find that $d h / d \Lambda<0$ for $\lambda_{M}<$ $k_{\gamma}<\sqrt{\left(-4 c+c^{2} \lambda_{M}^{-2}\right) / 32}$ and $d h / d \Lambda>0$ for $\sqrt{\left(-4 c+c^{2} \lambda_{M}^{-2}\right) / 32}<k_{\gamma}<\delta_{M}$. Since $(\sqrt{|c|} / 2) \tanh (\sqrt{|c|} r / 2)$ is monotone increasing with respect to $r$ and $\sqrt{|c|} \operatorname{coth} \sqrt{|c|} r$ is monotone decreasing with respect to $r$, if we take two tubes $T\left(r_{1}\right), T\left(r_{2}\right)\left(r_{1}>r_{2}\right)$, we find that $\mathscr{E}\left(T\left(r_{1}\right)\right)$ and $\mathscr{E}\left(T\left(r_{2}\right)\right)$ intersect with each other only at one point (see Figure 5). The $k$-coordinate $\varkappa\left(r_{1}, r_{2}\right)$ of this point satisfies $\left(-4 c+c^{2} \lambda_{T\left(r_{1}\right)}^{-2}\right) / 32<\chi\left(r_{1}, r_{2}\right)^{2}<\left(-4 c+c^{2} \lambda_{T\left(r_{2}\right)}^{-2}\right) / 32$. As $\mathscr{E}(T(r))$ converges to $\mathscr{E}(H S)$ when $r$ goes to infinity, we see that the intersection of $\mathscr{E}\left(T\left(r_{1}\right)\right)$ and $\mathscr{E}\left(T\left(r_{2}\right)\right)$ lies in $\mathscr{M \mathscr { B }}_{2}\left(\mathbf{C} H^{n}\right)$. Since $\lim _{r \downarrow 0}\left(-4 c+c^{2} \lambda_{T(r)}^{-2}\right) / 32=\lim _{r \downarrow 0} \delta_{T(r)}=$ $\infty$, we see that $\mathscr{M}_{2}\left(\mathbf{C} H^{n}\right)$ is covered by $\{\mathscr{E}(T(r)) \mid r>0\}$. We set $\mathscr{M} \mathscr{U}_{2}\left(\mathbf{C} H^{n}\right)$ $=\mathscr{M}_{2}\left(\mathbf{C} H^{n}\right) \backslash \mathscr{M} \mathscr{B}_{2}\left(\mathbf{C} H^{n}\right)$ and denote by $\mathscr{T}$ the set of all congruence classes of circles on $\mathrm{CH}^{n}(c)$ of geodesic curvature not greater than $\sqrt{|c|}$ and of complex torsion \pm 1 . As we have $\lim _{\Lambda \downarrow 0} h(\Lambda)=1$, and $\lim _{\Lambda \uparrow k_{\nu}^{2}} h(\Lambda)=0$ for each $k_{\gamma}$, and have $\lim _{r \downarrow 0} \lambda_{T(r)}=0$, we find that the set $\mathscr{M}_{2}\left(\mathbf{C} H^{n}\right) \backslash \mathscr{T}$ is covered by $\{\mathscr{E}(T(r)) \mid r>0\}$, and that $\left\{\mathscr{E}(T(r)) \cap \mathscr{M} \mathscr{U}_{2}\left(\mathbf{C} H^{n}\right) \mid r>0\right\}$ covers $\mathscr{M} \mathscr{U}_{2}\left(\mathbf{C} H^{n}\right) \backslash \mathscr{T}$. Summarizing up we have

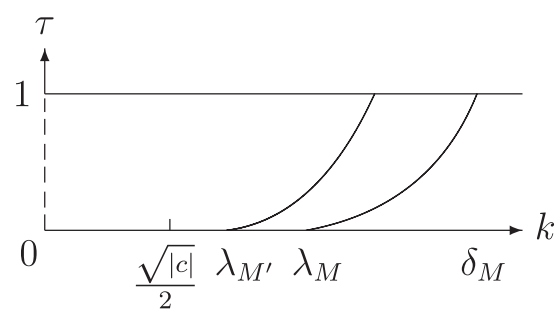

FIGURE 4. $\mathscr{E}(M), \mathscr{E}\left(M^{\prime}\right)$ for $M=G\left(r_{1}\right), M^{\prime}=$ $G\left(r_{2}\right)$ with $r_{1}<r_{2}$

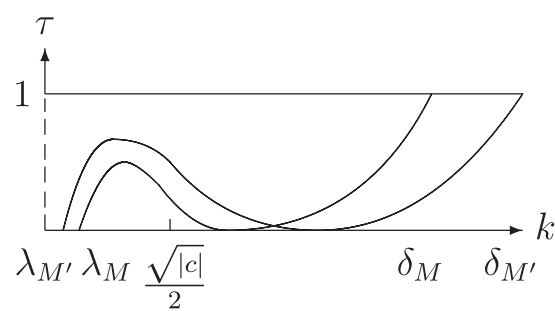

Figure 5. $\mathscr{E}(M), \mathscr{E}\left(M^{\prime}\right)$ for $M=T\left(r_{1}\right), M^{\prime}=$ $T\left(r_{2}\right)$ with $r_{1}>r_{2}$ 
THEOREM 1. (1) The family $\{\mathscr{E}(G(r)) \mid r>0\}$ forms a foliation of the moduli space $\mathscr{M \mathscr { B }}_{2}\left(\mathbf{C} H^{n}\right)$ of bounded circles on $\mathbf{C} H^{n}$.

(2) The family $\{\mathscr{E}(T(r)) \mid r>0\}$ covers the set $\mathscr{M}_{2}\left(\mathbf{C} H^{n}\right) \backslash \mathscr{T}$ of all congruence classes of circles except unbounded circles of complex torsion \pm 1 .

(3) Two distinct $\mathscr{E}\left(T\left(r_{1}\right)\right), \mathscr{E}\left(T\left(r_{2}\right)\right)$ intersect each other only at one point. This point lies in $\mathscr{M B}_{2}\left(\mathbf{C} H^{n}\right)$.

(4) The family $\left\{\mathscr{E}(T(r)) \cap \mathscr{M} \mathscr{U}_{2}\left(\mathbf{C} H^{n}\right) \mid r>0\right\} \quad$ forms a foliation of $\mathscr{M} \mathscr{U}_{2}\left(\mathbf{C} H^{n}\right) \backslash \mathscr{T}$.

In the above we studied the moduli space of extrinsic circular trajectories on each totally $\eta$-umbilic real hypersurface. Being concerned with Propositions 1 and 3 , in the following we shall study their moduli spaces for some families of totally $\eta$-umbilic real hypersurfaces, and investigate how circles of given geodesic curvature are obtained as extrinsic shapes of trajectories on some real hypersurfaces.

THEOREM 2. Every bounded circle of geodesic curvature $\sqrt{|c|} / 2<k \leq \sqrt{|c|}$ on a complex hyperbolic space $\mathbf{C} H^{n}(c)$ is the extrinsic shape of a trajectory for some Sasakian magnetic field on some geodesic sphere $G(r)$ of radius $r \geq(\log 3)$ / $\sqrt{|c|}$. The triplet of such a trajectory, a Sasakian magnetic field and a geodesic sphere is uniquely determined.

THEOREM 3. Every circle of geodesic curvature $0<k \leq \sqrt{|c|} / 2$ and of complex torsion $\tau \neq \pm 1$ on a complex hyperbolic space $\mathbf{C} H^{n}(c)$ is the extrinsic shape of a trajectory for some Sasakian magnetic field on some tube $T(r)$ around $\mathbf{C} H^{n-1}$. For such a circle with complex torsion $|\tau|<1$, the triplet of such a trajectory, a Sasakian magnetic field and a tube is uniquely determined.

One can easily guess these results by the first and the last assertions of Theorem 1, but in order to show clearly how trajectories correspond to circles we here give their proofs.

Proof of Theorems 2 and 3. We take a circle $\sigma$ on $\mathbf{C H}^{n}(c)$ satisfying one of the following:

i) $k_{\sigma} \leq \sqrt{|c|} / 2$ and $\tau_{\sigma} \neq \pm 1$

ii) $\sqrt{|c|} / 2<k_{\sigma} \leq \sqrt{|c|}$ and $\left|\tau_{\sigma}\right|<v\left(k_{\sigma}\right)$.

We denote by $\left\{\dot{\sigma}, Y_{\sigma}\right\}$ its Frenet frame. In other words, this $\sigma$ satisfies $\tilde{\nabla}_{\dot{\sigma}} \dot{\sigma}=$ $k_{\sigma} Y_{\sigma}$ and $\tilde{\nabla}_{\dot{\sigma}} Y_{\sigma}=-k_{\sigma} \dot{\sigma}$. By Lemma 2 and (4.2), we first solve the following system of equations on $\lambda$ and $\rho$

$$
\left\{\begin{array}{l}
16 \lambda^{4}+8|c| \rho^{2} \lambda^{2}+c^{2} \rho^{2}=16 k_{\sigma}^{2} \lambda^{2} \\
\rho\left(|c|-2|c| \rho^{2}-4 \lambda^{2}\right)=4 k_{\sigma} \tau_{\sigma} \lambda
\end{array}\right.
$$

As we need to get a solution with $|\rho| \leq 1$, the first equation of (4.4) shows that $\lambda \leq k_{\sigma} \leq \lambda+(|c| /(4 \lambda))$. Since $k_{\sigma} \leq \sqrt{|c|}$, the second inequality $k_{\sigma} \leq \lambda+$ 
$(|c| /(4 \lambda))$ automatically holds. We hence study (4.4) under the condition that $\lambda \leq k_{\sigma} \leq \sqrt{|c|}$. By substituting $\rho^{2}=16 \lambda^{2}\left(k_{\sigma}^{2}-\lambda^{2}\right) /\left(8|c| \lambda^{2}+c^{2}\right)$ into the second, we have

$$
\tau_{\sigma}^{2}=\frac{\left(k^{2}-\lambda^{2}\right)\left(4 c^{2} \lambda^{2}-c^{3}+32 c k_{\sigma}^{2} \lambda^{2}\right)^{2}}{k_{\sigma}^{2}\left(8|c| \lambda^{2}+c^{2}\right)^{3}} .
$$

We put the right hand side of the above equality as $f_{k_{\sigma}}(\lambda)$, and consider it a function of $\lambda$. We then find that $f_{k_{\sigma}}(\lambda)$ is monotone decreasing with respect to $\lambda$, and that $f_{k_{\sigma}}(k)=0, f_{k_{\sigma}}(0)=1$ and $f_{k_{\sigma}}(\sqrt{|c|} / 2)=\left(4 k_{\sigma}^{2}+c\right)^{3} /\left(27 c^{2} k_{\sigma}^{2}\right)=v\left(k_{\sigma}\right)^{2}$.

First we study the case that $\sqrt{|c|} / 2<k_{\sigma} \leq \sqrt{|c|}$ and $\left|\tau_{\sigma}\right|<v\left(k_{\sigma}\right)$. When we consider geodesic spheres, their principal curvatures for vectors orthogonal to characteristic vectors are in the interval $\sqrt{|c|} / 2<\lambda_{M} \leq \sqrt{|c|}$ if and only if their radii satisfy $r \geq(\log 3) / \sqrt{|c|}$. Thus for $\tau_{\sigma}$ with $0 \leq \tau_{\sigma}<v\left(k_{\sigma}\right)$ we have unique $\lambda$ satisfying $f_{k_{\sigma}}(\lambda)=\tau_{\sigma}^{2}$. This determines $\rho^{2}$. Hence (4.4) has a unique solution $(\lambda, \rho)$ in this case. By using this solution, we set $v=\left(4 k_{\sigma} \lambda Y_{\sigma}(0)-c \rho J \dot{\sigma}(0)\right) /$ $\left(4 \lambda^{2}-2 c \rho^{2}\right)$. As we have

$$
\begin{aligned}
\|v\|^{2}\left(4 \lambda^{2}-2 c \rho^{2}\right)^{2} & =\left\|4 k_{\sigma} \lambda Y_{\sigma}(0)-c \rho J \dot{\sigma}(0)\right\|^{2} \\
& =16 k_{\sigma}^{2} \lambda^{2}+8 c k_{\sigma} \tau_{\sigma} \lambda \rho+c^{2} \rho^{2} \\
& =16 \lambda^{4}-8 c \rho^{2} \lambda^{2}+c^{2} \rho^{2}+2 c \rho^{2}\left(-c+2 c \rho^{2}-4 \lambda^{2}\right)+c^{2} \rho^{2} \\
& =\left(4 \lambda^{2}-2 c \rho^{2}\right)^{2}
\end{aligned}
$$

we see $v$ is a unit tangent vector. We choose the geodesic $\alpha$ with initial vector $v$ and take a geodesic sphere $G$ of radius $r=(2 / \sqrt{|c|}) \operatorname{coth}^{-1}(2 \lambda / \sqrt{|c|})$ centered at $\alpha(r)$. Since its inward unit normal $\mathscr{N}$ satisfies $\mathscr{N}_{\sigma(0)}=v$ and $\dot{\sigma}(0)$ is orthogonal to $v$, we find that $\dot{\sigma}(0)$ is tangent to $G$ and we have

$$
\left\langle\dot{\sigma}(0),-J \mathscr{N}_{\sigma(0)}\right\rangle=\frac{-4 k_{\sigma} \tau_{\sigma} \lambda-c \rho}{4 \lambda^{2}-2 c \rho^{2}}=\rho .
$$

Thus if we take the trajectory $\gamma$ for $\mathbf{F}_{c \rho /(4 \lambda)}$ on $G$ satisfying $\dot{\gamma}(0)=\dot{\sigma}(0)$, its structure torsion is $\rho$, and hence we find by Lemma 2 and (4.4) that its extrinsic shape is a circle with

$$
k_{\gamma}=\sqrt{\frac{c^{2} \rho^{2}}{16 \lambda^{2}}-\frac{c \rho^{2}}{2}+\lambda^{2}}=k_{\sigma}, \quad \tau_{\gamma}=\frac{1}{k_{\gamma}}\left(\frac{c \rho^{3}}{2 \lambda}-\frac{c \rho}{4 \lambda}-\lambda \rho\right)=\tau_{\sigma} .
$$

Since the Frenet frame of the extrinsic shape of $\gamma$ at $\gamma(0)$ is $\left\{\dot{\sigma}(0), Y_{\gamma}(0)\right\}$ with

$$
Y_{\gamma}(0)=\frac{1}{k_{\gamma}}\left\{\frac{c \rho}{4 \lambda} J \dot{\sigma}(0)+\left(\lambda-\frac{c \rho^{2}}{2 \lambda}\right) v\right\}
$$


by (4.1), we find that the extrinsic shape of $\gamma$ coincides with $\sigma$. Thus we obtain that every bounded circle of geodesic curvature $\sqrt{|c|} / 2<k \leq \sqrt{|c|}$ on $\mathbf{C} H^{n}(c)$ is the extrinsic shape of a trajectory for some Sasakian magnetic field on some geodesic sphere of radius $r \geq \log (3 / \sqrt{|c|})$.

We now show that the above triplet $\left(G, \mathbf{F}_{c \rho /(4 \lambda)}, \gamma\right)$ is unique. If we suppose that the extrinsic shape of a trajectory $\varsigma$ for $\mathbf{F}_{\kappa}$ on a geodesic sphere $G(R)$ coincides with $\sigma$, then Lemma 2 and (4.1) show that

- $\kappa=-\left(|c| \rho_{\varsigma}\right) /\left(4 \lambda_{G(R)}\right)$

- the pair $\left(\lambda_{G(R)}, \rho_{\varsigma}\right)$ satisfies (4.4),

- $k_{\sigma} Y_{\sigma}=\kappa J \dot{\zeta}+\left(\lambda_{G(R)}+\left(|c| \rho_{\varsigma}^{2}\right) /\left(4 \lambda_{G(R)}\right)\right) \mathscr{N}_{G(R)}$ with the inward unit normal $\mathscr{N}_{G(R)}$ of $G(R)$.

Since (4.4) has a unique solution for given $k_{\sigma}, \tau_{\sigma}$, we see $\mathscr{N}_{G(R)}$ coincides with $v$ at $\sigma(0)$. Therefore we find $G(R)=G$, and hence get $\mathbf{F}_{\kappa}=\mathbf{F}_{c \rho /\left(4 \lambda_{G(R)}\right)}$ and $\varsigma=\gamma$. This completes the proof of Theorem 2 .

Next we study the case that $0<k_{\sigma} \leq \sqrt{|c|} / 2$ and $\left|\tau_{\sigma}\right|<1$. For tubes around totally geodesic $\mathbf{C} H^{n-1}$, principal curvatures of vectors orthogonal to characteristic vectors are in the interval $0<\lambda_{M}<\sqrt{|c|} / 2$. As we have unique $\lambda$ satisfying $f_{k_{\sigma}}(\lambda)=\tau_{\sigma}^{2}$, it determines $\rho^{2}$. Hence (4.4) has a unique solution $(\lambda, \rho)$ also in this case. Along the same lines as for the case of geodesic spheres, we get the assertion of Theorem 3 .

We can do the similar argument for the case $k_{\sigma}>\sqrt{|c|}$. In this case the inequalities $\lambda \leq k_{\sigma} \leq \lambda+(|c| /(4 \lambda))$ leads us to $\left(k_{\sigma}+\sqrt{k_{\sigma}^{2}+c}\right) / 2 \leq \lambda \leq k_{\sigma}$. We therefore obtain that every bounded circle on $\mathbf{C H}^{n}(c)$ is the extrinsic shape of a trajectory for some Sasakian magnetic field on some geodesic sphere, and that for such a circle with complex torsion $|\tau|<1$ the triplet of a trajectory, a Sasakian magnetic field and a geodesic sphere is uniquely determined ([5]). As we have $5 \sqrt{|c|} / 4 \geq \delta_{M}\left(=\lambda_{M}+\left(|c| /\left(4 \lambda_{M}\right)\right)\right)$ if and only if the radius of $M$ satisfies $r \geq(\log 3) / \sqrt{|c|}$, we obtain

Proposition 4. Every circle of geodesic curvature $k>5 \sqrt{|c|} / 4$ on $\mathbf{C H}(c)$ $i$ the extrinsic shape of a trajectory for some Sasakian magnetic field on some geodesic sphere $G(r)$ of positive curvature.

We should note that bounded circles are also extrinsic shapes of trajectories for some Sasakian magnetic fields on tubes around totally geodesic $\mathbf{C} H^{n-1}$.

THEOREM ([5]). Every circle on $\mathbf{C} H^{n}(c)$ except circles of geodesic curvature $0<k \leq \sqrt{|c|}$ and of complex torsion $\tau= \pm 1$ is the extrinsic shape of a trajectory for some Sasakian magnetic field on some tube $T(r)$. When a circle is unbounded, such a triplet of a trajectory, a Sasakian magnetic field, and a tube is uniquely determined. When a circle is bounded, exactly two triplets correspond to it.

We have a result corresponding to Theorem 2 . 
Proposition 5. Every bounded circle of geodesic curvature $\sqrt{|c|} / 2<k \leq$ $\sqrt{|c|}$ on a complex hyperbolic space $\mathbf{C} H^{n}(c)$ is the extrinsic shape of a trajectory for some Sasakian magnetic field on some tube $T(r)$. The triplet of such a trajectory, a Sasakian magnetic field and a geodesic sphere is uniquely determined.

If we consider the condition $\delta_{M} \leq 5 \sqrt{|c|} / 4$ for tubes around totally geodesic complex hypersurfaces, we get the following.

Proposition 6. Every circle of geodesic curvature $k \geq 5 \sqrt{|c|} / 4$ on a complex hyperbolic space $\mathbf{C H}^{n}(c)$ is the extrinsic shape of a trajectory for some Sasakian magnetic field on some tube $T(r)$ of radius $r \leq(\log 3) / \sqrt{|c|}$.

\section{A characterization of tubes around complex hypersurfaces}

Since Kajiwara-Maeda's result shows a characterization of geodesic spheres of small radius, we here consider to give a characterization of tubes around totally geodesic complex hypersurfaces.

We here consider the following condition on the characteristic vector $\xi_{p}$ at $p \in M$ :

(ET) The extrinsic shape of the trajectory $\gamma_{0}$ for some Sasakian magnetic field $\mathbf{F}_{\kappa_{0}}$ with $\dot{\gamma}_{0}(0)=\xi_{p}$ is a circle of geodesic curvature $k \neq\left|\kappa_{0}\right|$.

THEOREM 4. A connected real hypersurface $M$ in $\mathbf{C H}^{n}(c)$ is locally congruent to a tube $T(r)$ around totally geodesic complex hypersurface $\mathbf{C} H^{n-1}$ if and only if it satisfies the following conditions at each point $p \in M$ :

i) The condition (ET) holds at $p$;

ii) There exist constants $\kappa_{p}, \rho_{p}$ with $\kappa_{p} \neq 0,\left|\rho_{p}\right|<1$ and linearly independent unit tangent vectors $v_{1}, \ldots, v_{2 n-2} \in U_{p} M$ with $\left\langle v_{i}, \xi_{p}\right\rangle=\rho_{p}$ which satisfy that the extrinsic shapes of trajectories $\gamma_{i}(i=1, \ldots, 2 n-2)$ for $\mathbf{F}_{\kappa_{p}}$ with $\dot{\gamma}_{i}(0)=v_{i}$ are circles of geodesic curvature $k_{i}$ with $k_{i}<\sqrt{|c|} / 2$ and $k_{i} \neq\left|\kappa_{p}\right|$.

Proof. By Lemma 2, we are enough to show the "if" part. For a trajectory $\gamma$ for $\mathbf{F}_{\kappa}$ on $M$ we have

$$
\begin{aligned}
& \tilde{\nabla}_{\dot{\gamma}} \dot{\gamma}=\nabla_{\dot{\gamma}} \dot{\gamma}+\left\langle A_{M} \dot{\gamma}, \dot{\gamma}\right\rangle \mathscr{N}=\kappa J \dot{\gamma}+\left(\left\langle A_{M} \dot{\gamma}, \dot{\gamma}\right\rangle-\kappa \rho\right) \mathscr{N}, \\
& \tilde{\nabla}_{\dot{\gamma}} \tilde{\nabla}_{\dot{\gamma}} \dot{\gamma}=-\kappa^{2} \dot{\gamma}-\left(\left\langle A_{M} \dot{\gamma}, \dot{\gamma}\right\rangle-\kappa \rho\right)\left(A_{M} \dot{\gamma}+\kappa \xi\right)+\frac{d}{d t}\left(\left\langle A_{M} \dot{\gamma}, \dot{\gamma}\right\rangle-\kappa \rho\right) \mathscr{N} .
\end{aligned}
$$

Since $\gamma$ is parameterized by its arclength, the extrinsic shape of $\gamma$ is a circle of geodesic curvature $k_{\gamma}$ if and only if it satisfies $\tilde{\nabla}_{\dot{\gamma}} \tilde{\nabla}_{\dot{\gamma}} \dot{\gamma}=-k_{\gamma}^{2} \dot{\gamma}$. By the first condition, we have from (5.1) and (5.2) that

$$
k_{\gamma_{0}}=\left|\left\langle A_{M} \xi_{p}, \xi_{p}\right\rangle\right| \quad \text { and } \quad k_{\gamma_{0}}^{2} \xi_{p}=\left(\left\langle A_{M} \xi_{p}, \xi_{p}\right\rangle-\kappa_{0}\right) A_{M} \xi_{p}+\kappa_{0}\left\langle A_{M} \xi_{p}, \xi_{p}\right\rangle \xi_{p} .
$$


As $k_{\gamma_{0}} \neq\left|\kappa_{0}\right|$, we find that $\xi_{p}$ is a principal curvature vector. We denote by $\delta_{p}$ the principal curvature associated with $\xi_{p}$. By Lemma 1 it is locally constant with respect to $p$. As $M$ is connected we find it is constant, hence we can denote by $\delta_{M}$.

By the second condition we have from (5.2) that

$$
\begin{aligned}
\left(k_{i}^{2}-\kappa_{p}^{2}\right) \rho= & \left(\left\langle A_{M}\left(v_{i}-\rho_{p} \xi_{p}\right), v_{i}-\rho_{p} \xi_{p}\right\rangle\right. \\
& \left.+\delta_{M} \rho_{p}^{2}-\kappa_{p} \rho_{p}\right)\left(\rho_{p} \delta_{p}+\kappa_{p}\right), \\
\left(k_{i}^{2}-\kappa_{p}^{2}\right)\left(v-\rho_{p} \xi_{p}\right)= & \left(\left\langle A_{M}\left(v_{i}-\rho_{p} \xi_{p}\right), v_{i}-\rho_{p} \xi_{p}\right\rangle\right. \\
& \left.+\delta_{M} \rho_{p}^{2}-\kappa_{p} \rho_{p}\right) A_{M}\left(v_{i}-\rho_{p} \xi_{p}\right) .
\end{aligned}
$$

Since $k_{i} \neq\left|\kappa_{p}\right|$, by (5.4) we find that $v_{i}-\rho_{p} \xi_{p}$ is principal. We denote by $\alpha_{i}$ the principal curvature of $v_{i}-\rho_{p} \xi_{p}$. Then (5.3) and (5.4) turn to

$$
\begin{aligned}
\left(k_{i}^{2}-\kappa_{p}^{2}\right) \rho_{p} & =\left(\alpha_{i}\left(1-\rho_{p}^{2}\right)+\rho_{p}^{2} \delta_{p}-\kappa_{p} \rho_{p}\right)\left(\rho_{p} \delta+\kappa_{p}\right), \\
k_{i}^{2}-\kappa_{p}^{2} & =\left(\alpha_{i}\left(1-\rho_{p}^{2}\right)+\rho_{p}^{2} \delta_{p}-\kappa_{p} \rho_{p}\right) \alpha_{i} .
\end{aligned}
$$

Hence we have either $\rho_{p} \alpha_{i}=\rho_{p} \delta_{p}+\kappa_{p}$ or $\alpha_{i}\left(1-\rho_{p}^{2}\right)+\rho_{p}^{2} \delta_{p}-\kappa_{p} \rho_{p}=0$ holds. If $\rho_{p}=0$, we have $\alpha_{i}^{2}=k_{i}^{2}-\kappa_{p}^{2}$ and $\alpha_{i} \kappa_{p}=0$ by (5.5) and (5.6). Since $k_{i} \neq|\kappa|$ we see $\kappa_{p}=0$, which is a contradiction. Thus we have $\rho_{p} \neq 0$. Again as $k_{i} \neq\left|\kappa_{p}\right|$, we find $\alpha_{i}\left(1-\rho_{p}^{2}\right)+\rho_{p}^{2} \delta_{p}-\kappa_{p} \rho_{p} \neq 0$ and obtain $\alpha_{i}=\delta_{p}+\left(\kappa_{p} / \rho_{p}\right)$. Thus, we have $\alpha_{1}=\cdots=\alpha_{2 n-2}\left(=\alpha_{p}\right)$, hence $M$ is totally $\eta$-umbilic.

We have by (5.1) that

$$
k_{i}^{2}=\kappa_{p}^{2}\left(1-\rho_{p}^{2}\right)+\left\langle A \dot{\gamma}_{i}, \dot{\gamma}_{i}\right\rangle^{2}=\kappa_{p}^{2}\left(1-\rho_{p}^{2}\right)+\left\{\alpha_{p}+\left(\delta_{p}-\alpha_{p}\right) \rho_{p}^{2}\right\}^{2} .
$$

For a totally $\eta$-umbilic real hypersurface $M$ in $\mathbf{C} H^{n}$ we see

$$
\delta_{M}-\lambda_{M}= \begin{cases}\sqrt{|c|} / 2, & \text { when } M=H S, \\ (\sqrt{|c|} / 2) \tanh (\sqrt{|c|} r / 2), & \text { when } M=G(r), \\ (\sqrt{|c|} / 2) \operatorname{coth}(\sqrt{|c|} r / 2), & \text { when } M=T(r) .\end{cases}
$$

Thus we have $\delta_{M}-\lambda_{M}>0$, hence find that $k_{i}>\lambda_{M}$. As geodesic curvatures of extrinsic shape of trajectories are less than $\sqrt{|c|} / 2$, we see $M$ is congruent to a tubes around totally geodesic complex hypersurface.

Next we study a condition concerning the strength of a Sasakian magnetic field and the geodesic curvature of extrinsic shapes.

THEOREM 5. A connected real hypersurface $M$ in $\mathbf{C} H^{n}(c)$ is locally congruent to a tube $T(r)$ around totally geodesic complex hypersurface $\mathbf{C} H^{n-1}$ if and only if it satisfies the following conditions with some constants $\kappa, k$ satisfying $k \neq|\kappa|$ and $0<k<\sqrt{|c|} / 2$ : 
i) The condition (ET) holds at each point $p \in M$;

ii) There exist linearly independent tangent vectors $v_{1}, \ldots, v_{2 n-2} \in U_{p} M$ satisfying that

(a) $v_{i} \neq \pm \xi_{p}$ for $i=1, \ldots, 2 n-2$

(b) the extrinsic shapes of trajectories $\gamma_{i}(i=1, \ldots, 2 n-2)$ for $\mathbf{F}_{\kappa}$ with $\dot{\gamma}_{i}(0)=v_{i}$ are circles of common geodesic curvature $k$.

Proof. We are enough to show the "if" part. Along the same lines as in the proof of Theorem 4, we see $M$ is a Hopf hypersurface. We denote by $\delta_{M}$ the principal curvature associated with $\xi$. We put $\rho_{i}=\left\langle v_{i}, \xi_{p}\right\rangle$. As $k \neq|\kappa|$, we find by the proof of Theorem 4 that each $v_{i}-\rho_{i} \xi_{p}$ is principal and that $\alpha_{i} \rho_{i}=\delta_{M} \rho_{i}+\kappa$, where $\alpha_{i}$ is the principal curvature of $v_{i}-\rho_{i} \xi_{p}$.

When $\rho_{i}=0$, we have $\kappa=0$. As

$$
k^{2}=\kappa^{2}\left(1-\rho_{i}^{2}\right)+\left\{\alpha_{i}+\left(\delta_{M}-\alpha_{i}\right) \rho_{i}^{2}\right\}^{2},
$$

this shows that $\alpha_{i}= \pm k$. When $\rho_{i} \neq 0$, substituting $\alpha_{i}=\delta_{M}+\left(\kappa / \rho_{i}\right)$ into (5.7), we find that $\rho_{i}$ satisfies the following equation

$$
-2 \kappa \delta_{M} \rho_{i}^{3}+\left(\delta_{M}-k^{2}-\kappa^{2}\right) \rho_{i}^{2}+2 \kappa \delta_{M} \rho_{i}+\kappa^{2}=0 .
$$

Thus $\rho_{i}$ is one of the three solutions of this cubic equation. Therefore, by perturbation theory ([11]) we find that each $\alpha_{i}$ is locally constant. This means that $M$ is a Hopf real hypersurface all of whose principal curvatures are constant.

We check that whether homogeneous Hopf hypersurfaces satisfy the condition. When $M$ is one of $H S, G(r), T_{\ell}(r)$ we find by their principal curvatures $\delta_{M}, \lambda_{M}$ and by (5.7) that $k>\lambda_{M}>\sqrt{|c|} / 2$. Hence these do not satisfy the condition. We study the case $M=R(r)$. In this case we have

$$
\lambda_{M}=\sqrt{|c|} v / 2, \quad \mu_{M}=\sqrt{|c|} /(2 v), \quad \delta_{M}=2 \sqrt{|c|} /\left(v+v^{-1}\right)
$$

with $v=\cosh (\sqrt{|c|} r / 2)$. We have $\delta_{M} \neq \mu_{M}$. When $v=\sqrt{3}$, we have $\delta_{M}=\lambda_{M}$. Hence $\kappa=0$ and $k=\delta_{M}$. Thus, for $i$ with $\alpha_{i}=\mu_{M}$ we have $\rho_{i}=0$. Therefore we get $k^{2}=\mu_{M}^{2}\left(\neq \lambda_{M}^{2}\right)$, which is a contradiction. When $v \neq \sqrt{3}$, we have $\delta_{M} \neq \lambda_{M}$. Therefore we have $\rho_{i}=\kappa /\left(\delta_{M}-\alpha_{i}\right)$ hence get

$$
k^{2}=\kappa^{2}\left\{1-\frac{\kappa^{2}}{\left(\delta_{M}-\alpha_{i}\right)^{2}}\right\}+\left\{\alpha_{i}+\frac{\kappa^{2}}{\delta_{M}-\alpha_{i}}\right\}^{2}=\kappa^{2}+\alpha_{i}^{2}+\frac{2 \kappa^{2} \alpha_{i}}{\delta_{M}-\alpha_{i}} .
$$

As $k<\sqrt{|c|} / 2$, we need $\delta_{M}-\lambda_{M}<0$, hence we have $v>\sqrt{3}$. Moreover, we have

$$
\frac{|c| v^{2}}{4}+\frac{2 \kappa^{2}\left(v^{2}+1\right)}{\left(3-v^{2}\right)}=\frac{|c|}{4 v^{2}}+\frac{2 \kappa^{2}\left(v^{2}+1\right)}{\left(3 v^{2}-1\right)}=k^{2}-\kappa^{2},
$$


which leads us to $\kappa^{2}=|c|\left(v^{2}-3\right)\left(3-v^{-2}\right) / 32$. Here, as $\kappa+\left(\delta_{M}-\alpha_{i}\right) \rho_{i}=0$ and $\left|\rho_{i}\right|<1$, we have $|\kappa|<\min \left\{\left|\delta_{M}-\lambda_{M}\right|,\left|\delta_{M}-\mu_{M}\right|\right\}$. Therefore we obtain

$$
\left\{\begin{array}{l}
\left(3-v^{-2}\right)\left(v+v^{-1}\right)^{2}<8\left(v^{2}-3\right), \\
\left(v^{2}-3\right)\left(v+v^{-1}\right)^{2}<8\left(3-v^{-2}\right),
\end{array}\right.
$$

which are equivalent to

$$
\left\{\begin{array}{l}
\left(5 v^{2}+1\right)\left(v^{4}-3 v^{2}+1\right)>0 \\
\left(v^{2}+5\right)\left(v^{4}-3 v^{2}+1\right)<0
\end{array}\right.
$$

This is also a contradiction. Hence $R(r)$ does not satisfy the condition. Thus we get the conclusion.

\section{REFERENCES}

[ 1 ] T. AdACHI, Kähler magnetic flows on a manifold of constant holomorphic sectional curvature, Tokyo J. Math. 18 (1995), 473-483.

[2] T. Adachi, Trajectories on geodesic spheres in a non-flat complex space form, J. Geom. 90 (2008), 1-29.

[3] T. AdAchi, Dynamical systematic aspect on horocyclic circles in a complex hyperbolic space, Prospects of Differential Geometry and its Related Fields (T. Adachi, H. Hashimoto and M. Hristov eds.), World Scientific, Singapore, 2013, 99-114.

[4] T. AdAchI, Extrinsic circular trajectories on geodesic spheres in a complex projective space, Differential Geometry of Submanifolds and its Related Topics (S. Maeda, Y. Ohnita and Q.-M. Cheng eds), World Scientific, Singapore, 2013, 44-59.

[5] T. AdAchi, Foliation on the moduli space of extrinsic circular trajectories on a complex hyperbolic space, Topology and its Applications 196 (2015), 311-324.

[6] T. AdACHI AND S. MaEdA, Global behaviors of circles in a complex hyperbolic space, Tsukuba J. Math. 21 (1997), 29-42.

[7] T. Adachi, S. Maeda and M. Yamagishi, Length spectrum of geodesic spheres in a nonflat complex space form, J. Math. Soc. Japan 54 (2002), 373-408.

[8] T. BAO AND T. ADACHI, Circular trajectories on real hypersurfaces in a nonflat complex space form, J. Geom. 96 (2009), 41-55.

[9] J. BERNDT, Real hypersurfaces with constant principal curvatures in complex hyperbolic space, J. Reine Angew. Math. 395 (1989), 132-141.

[10] A. Cомтет, On the Landau levels on the hyperbolic plane, Ann. Phys. 173 (1987), 185-209.

[11] T. Kato, Perturbation theory for linear operators, Springer, 1966.

[12] T. KaJiwara And S. MaEda, Sectional curvatures of geodesic spheres in a complex hyperbolic space, Kodai Math. J. 38 (2015), 604-619.

[13] U.-H. Ki AND Y. J. SuH, On real hypersurfaces of a complex space form, Math. J. Okayama Univ. 32 (1990), 207-221.

[14] Y. MaedA, On real hypersurfaces of a complex projective space, J. Math. Soc. Japan 28 (1976), 529-540.

[15] S. Maeda And T. Adachi, Sasakian curves on hypersurfaces of type (A) in a nonflat complex space form, Results Math. 56 (2009), 489-499.

[16] S. MaEdA, T. AdACHI AND Y. H. KIM, Characterizations of geodesic hyperspheres in a nonflat complex space form, Glasgow Math. J. 55 (2013), 217-227. 
[17] S. Maeda and Y. Ohnita, Helical geodesic immersion into complex space form, Geom. Dedicata 30 (1989), 93-114.

[18] T. SunAda, Magnetic flows on a Riemann surface, Proc. KAIST Math. Workshop 8 (1993), 93-108.

\section{Tuya Bao}

College of Mathematics

InNer Mongolia University fOR the Nationalities

TongliaO, InNer Mongolia 028043

P. R. CHINA

E-mail: baotuya1981@126.com

Toshiaki Adachi

Department of Mathematics

Nagoya Institute of Technology

NAGOYa 466-8555

JAPAN

E-mail: adachi@nitech.ac.jp 